\title{
GROWTH OF MEROMORPHIC SOLUTIONS OF GENERAL COMPLEX LINEAR DIFFERENTIAL-DIFFERENCE EQUATION
}

\author{
Zhen Chen, Xiu-Min Zheng
}

Abstract. In this paper, we investigate the ralations between the growth of entire or meromorphic coefficients and the growth of meromorphic solutions of general complex linear differential-difference equation, and obtain the lower bound of the order of meromorphic solutions by comparing the (lower) orders or the (lower) types of the coefficients. Our results can be seen as generalizations for both the case of complex linear differential equation and the case of complex linear difference equation.

2010 Mathematics Subject Classification: 30D35; 39B32.

Keywords: Complex linear differential-difference equation, meromorphic solution, (lower) order, (lower) type.

\section{INTRODUCTION AND MAIN RESULTS}

Throughout this paper, we assume that $f(z)$ is a meromorphic function in the whole complex plane, and use standard notations, such as $m(r, f), T(r, f), M(r, f)$, in the classic Nevanlinna theory ${ }^{[5,7,13]}$. And we also use the notations $\sigma(f)$ and $\mu(f)$ to denote respectively the order and the lower order of $f(z)$. Moreover, we give the definitions of the type and the lower type as follows.

Definition 1. (see[5,6]) Let $f(z)$ be a meromorphic function with the order $\sigma(f)(0<$ $\sigma(f)<\infty)$ and the lower order $\mu(f)(0<\mu(f)<\infty)$, then the type and the lower type of $f(z)$ are defined respectively by

$$
\tau(f)=\varlimsup_{r \rightarrow \infty} \frac{T(r, f)}{r^{\sigma(f)}} \quad \text { and } \quad \underline{\tau}(f)=\lim _{r \rightarrow \infty} \frac{T(r, f)}{r^{\mu(f)}} .
$$

If $f(z)$ is also an entire function, then we denote

$$
\tau_{M}(f)=\varlimsup_{r \rightarrow \infty} \frac{\log M(r, f)}{r^{\sigma(f)}} \quad \text { and } \quad \underline{\tau}_{M}(f)=\lim _{r \rightarrow \infty} \frac{\log M(r, f)}{r^{\mu(f)}} .
$$


Zhen Chen, Xiu-Min Zheng - Meromorphic solutions of differential-difference ...

Many scholars applied Nevanlinna theory and its difference analogues to study the properties of meromorphic solutions of complex linear differential equation

$$
f^{(n)}+A_{n-1}(z) f^{(n-1)}+\cdots+A_{1}(z) f^{\prime}+A_{0}(z) f=0
$$

and complex linear difference equation

$$
A_{n}(z) f\left(z+c_{n}\right)+\cdots+A_{1}(z) f\left(z+c_{1}\right)+A_{0}(z) f(z)=0,
$$

and obtained fruitful results (see e.g. $[1,2,8,9,11,12,14]$ ). Interestingly, we find there are many similar properties for both the case of complex linear differential equation (1) and the case of complex linear difference equation (2). We give examples on this topic as follows.

For the case of complex linear differential equation (1), Wu and Zheng [12] weakened the normal condition (see e.g. [1,11]) that only one dominant coefficient of (1) has the (lower) order or the (lower) type strictly greater than the order or the type of other coefficients, and obtained the following Theorems 1 and 2 .

Theorem 1. (see[12]) Let $A_{i}(z), i=0,1, \cdots, n-1$ be entire functions and $j \in$ $\{1,2, \cdots, n-1\}$. If the following three assumptions hold simultaneously:

(1) $\max \left\{\mu_{p}\left(A_{j}\right), \sigma_{p}\left(A_{i}\right), i \neq 0, j\right\}=\sigma \leq \mu_{p}\left(A_{0}\right)<\infty, \mu_{p}\left(A_{0}\right)>0$;

(2) $\underline{\tau}_{p}\left(A_{0}\right)>\underline{\tau}_{p}\left(A_{j}\right)$, when $\mu_{p}\left(A_{0}\right)=\mu_{p}\left(A_{j}\right)$;

(3) $\underline{\tau}_{p}\left(A_{0}\right)>\max \left\{\tau_{p}\left(A_{i}\right): \sigma_{p}\left(A_{i}\right)=\mu_{p}\left(A_{0}\right), i \neq 0, j\right\}$, when $\mu_{p}\left(A_{0}\right)=\max \left\{\sigma_{p}\left(A_{i}\right)\right.$, $i \neq 0, j\}$.

Then any solution $f(z)(\not \equiv 0)$ of $(1)$ satisfies $\sigma_{p+1}(f) \geq \mu_{p}\left(A_{0}\right)$.

Theorem 2. (see[12]) Let $A_{i}(z), i=0,1, \cdots, n-1$ be entire functions and $j \in$ $\{1,2, \cdots, n-1\}$. If the following three assumptions hold simultaneously:

(1) $\max \left\{\mu_{p}\left(A_{0}\right), \sigma_{p}\left(A_{i}\right), i \neq 0, j\right\}=\delta \leq \mu_{p}\left(A_{j}\right)<\infty, \mu_{p}\left(A_{j}\right)>0$;

(2) $\underline{\tau}_{p}\left(A_{j}\right)>\underline{\tau}_{p}\left(A_{0}\right)$, when $\mu_{p}\left(A_{j}\right)=\mu_{p}\left(A_{0}\right)$;

(3) $\underline{\tau}_{p}\left(A_{j}\right)>\max \left\{\tau_{p}\left(A_{i}\right): \sigma_{p}\left(A_{i}\right)=\mu_{p}\left(A_{j}\right), i \neq 0, j\right\}$, when $\mu_{p}\left(A_{j}\right)=\max \left\{\sigma_{p}\left(A_{i}\right)\right.$, $i \neq 0, j\}$.

Then any transcendental solution $f(z)$ of (1) satisfies $\max \left\{\sigma_{p+1}(f), \lambda_{p}(f)\right\} \geq \mu_{p}\left(A_{j}\right)$, and the non-transcendental solution can only be polynomials that have degree no more than $j-1$.

For the case of complex linear difference equation (2), Luo and Zheng [9] obtained the similar results as Theorems 1 and 2, which also generalize the acknowledged good results (see e.g. $[2,8]$ ) in the field of complex linear difference equations.

Theorem 3. (see[9]) Let $A_{j}(z), j=0,1, \cdots, n$ be entire functions and $k, l(\neq k) \in$ $\{0,1, \cdots, n\}$. If the following three assumptions hold simultaneously: 
Zhen Chen, Xiu-Min Zheng - Meromorphic solutions of differential-difference ...

(1) $\max \left\{\mu\left(A_{k}\right), \sigma\left(A_{j}\right), j \neq k, l\right\}=\sigma \leq \mu\left(A_{l}\right)<\infty, \mu\left(A_{l}\right)>0$;

(2) $\underline{\tau}_{M}\left(A_{l}\right)>\underline{\tau}_{M}\left(A_{k}\right)$, when $\mu\left(A_{l}\right)=\mu\left(A_{k}\right)$;

(3) $\underline{\tau}_{M}\left(A_{l}\right)>\max \left\{\tau_{M}\left(A_{j}\right): \sigma\left(A_{j}\right)=\mu\left(A_{l}\right), j \neq k, l\right\}$, when $\mu\left(A_{l}\right)=\max \left\{\sigma\left(A_{j}\right)\right.$, $j \neq k, l\}$.

Then any meromorphic solution $f(z)(\not \equiv 0)$ of (2) satisfies $\sigma(f) \geq \mu\left(A_{l}\right)+1$.

Theorem 4. (see[9]) Let $A_{j}(z), j=0,1, \cdots, n$ be meromorphic functions and $k, l(\neq$ $k) \in\{0,1, \cdots, n\}$. If the following four assumptions hold simultaneously:

(1) $\max \left\{\mu\left(A_{k}\right), \sigma\left(A_{j}\right), j \neq k, l\right\}=\sigma \leq \mu\left(A_{l}\right)<\infty, \mu\left(A_{l}\right)>0$;

(2) $\underline{\tau}\left(A_{l}\right)>\underline{\tau}\left(A_{k}\right)$, when $\mu\left(A_{l}\right)=\mu\left(A_{k}\right)$;

(3) $\underline{\tau}\left(A_{l}\right)>\max \left\{\tau\left(A_{j}\right): \sigma\left(A_{j}\right)=\mu\left(A_{l}\right), j \neq k, l\right\}$, when $\mu\left(A_{l}\right)=\max \left\{\sigma\left(A_{j}\right), j \neq\right.$ $k, l\}$;

(4) $\delta\left(\infty, A_{l}\right)=\delta>0$.

Then any meromorphic solution $f(z)(\not \equiv 0)$ of (2) satisfies $\sigma(f) \geq \mu\left(A_{l}\right)+1$.

Remark 1. By a careful examination of the proof of Theorem 4, we note that " $\underline{\tau}\left(A_{l}\right)$ " in Theorem 4 should be replaced by " $\underline{\underline{\tau}}\left(A_{l}\right)$ ".

Inspired by the above results, we proceed to investigate the more general case than (1) and (2), that is, complex linear differential-difference equation

$$
\sum_{i=0}^{n} \sum_{j=0}^{m} A_{i j}(z) f^{(j)}\left(z+c_{i}\right)=0 .
$$

This is an interesting and meaningful topic. Not only for the sake that (3) is a combination of (1) and (2), and consequently inherits some common properties from (1) and (2). But also for the sake that (3) has its own application fields distinct from (1) and (2), and consequently (3) is of importance. There are already some results on this topic (see e.g. $[10,15]$ ). As generalizations of Theorems 1-4, we obtain the following two results in the filed of complex linear differential-difference equations.

Theorem 5. Let $A_{i j}(z), i=0,1, \cdots, n, j=0,1, \cdots m$ be entire functions, and $a, l \in\{0,1, \cdots, n\}, b \in\{0,1, \cdots, m\}$ such that $(a, b) \neq(l, 0)$. If the following three assumptions hold simultaneously:

(1) $\max \left\{\mu\left(A_{a b}\right), \sigma\left(A_{i j}\right),(i, j) \neq(a, b),(l, 0)\right\}=\sigma \leq \mu\left(A_{l 0}\right)<\infty, \mu\left(A_{l 0}\right)>0$;

(2) $\underline{\tau}_{M}\left(A_{l 0}\right)>\underline{\tau}_{M}\left(A_{a b}\right)$, when $\mu\left(A_{l 0}\right)=\mu\left(A_{a b}\right)$;

(3) $\underline{\tau}_{M}\left(A_{l 0}\right)>\max \left\{\tau_{M}\left(A_{i j}\right): \sigma\left(A_{i j}\right)=\mu\left(A_{l 0}\right),(i, j) \neq(a, b),(l, 0)\right\}$, when $\mu\left(A_{l 0}\right)=\max \left\{\sigma\left(A_{i j}\right),(i, j) \neq(a, b),(l, 0)\right\}$.

Then any meromorphic solution $f(z)(\not \equiv 0)$ of (3) satisfies $\sigma(f) \geq \mu\left(A_{l 0}\right)+1$.

Theorem 6. Let $A_{i j}(z), i=0,1, \cdots, n, j=0,1, \cdots m$ be meromorphic functions, and $a, l \in\{0,1, \cdots, n\}, b \in\{0,1, \cdots, m\}$ such that $(a, b) \neq(l, 0)$. If the following four assumptions hold simultaneously: 
Zhen Chen, Xiu-Min Zheng - Meromorphic solutions of differential-difference ...

(1) $\delta\left(\infty, A_{l 0}\right)=\delta>0$;

(2) $\max \left\{\mu\left(A_{a b}\right), \sigma\left(A_{i j}\right),(i, j) \neq(a, b),(l, 0)\right\}=\sigma \leq \mu\left(A_{l 0}\right)<\infty, \mu\left(A_{l 0}\right)>0$;

(3) $\delta \underline{\tau}\left(A_{l 0}\right)>\underline{\tau}\left(A_{a b}\right)$, when $\mu\left(A_{l 0}\right)=\mu\left(A_{a b}\right)$;

(4) $\delta \underline{\tau}\left(A_{l 0}\right)>\max \left\{\tau\left(A_{i j}\right): \sigma\left(A_{i j}\right)=\mu\left(A_{l 0}\right),(i, j) \neq(a, b),(l, 0)\right\}$, when $\mu\left(A_{l 0}\right)=$ $\max \left\{\sigma\left(A_{i j}\right),(i, j) \neq(a, b),(l, 0)\right\}$.

Then any meromorphic solution $f(z)(\not \equiv 0)$ of (3) satisfies $\sigma(f) \geq \mu\left(A_{l 0}\right)+1$.

Remark 2. The assumption (1) in Theorem 6 can be replaced by one of $\lambda\left(\frac{1}{A_{l 0}}\right)<$ $\mu\left(A_{l 0}\right)$, or $N\left(r, A_{l 0}\right)=o\left(m\left(r, A_{l 0}\right)\right)(r \rightarrow \infty)$, or $\mu\left(A_{l 0}\right)=\lim _{r \rightarrow \infty} \frac{\log m\left(r, A_{l 0}\right)}{\log r}$.

Remark 3. It is obvious that Theorems 1-4 are special cases of Theorems 5 and 6.

\section{LEMMAS FOR PROOFS OF MAIN RESULTS}

Lemma 7. (see[2]) Let $f(z)$ be a meromorphic function, $\eta(\neq 0), \eta_{1}, \eta_{2}\left(\eta_{1} \neq \eta_{2}\right)$ be arbitrary complex numbers, and let $\gamma(>1)$ and $\varepsilon(>0)$ be given real constants. Then there exists a subset $E \subset(1,+\infty)$ with finite logarithmic measure,

(1) and a constant $A$ depending only on $\gamma$ and $\eta$, such that for all $|z|=r \notin$ $[0,1] \cup E$, we have

$$
|\log | \frac{f(z+\eta)}{f(z)}|| \leq A\left(\frac{T(\gamma r, f)}{r}+\frac{n(\gamma r)}{r} \log ^{\gamma} r \log ^{+} n(\gamma r)\right) ;
$$

(2) and if in addition that $f(z)$ has finite order $\sigma$, and such that for all $|z|=r \notin$ $[0,1] \cup E$, we have

$$
\exp \left\{-r^{\sigma-1+\varepsilon}\right\} \leq\left|\frac{f\left(z+\eta_{1}\right)}{f\left(z+\eta_{2}\right)}\right| \leq \exp \left\{r^{\sigma-1+\varepsilon}\right\}
$$

Lemma 8. (see[4]) (1) Let $f(z)$ be a transcendental meromorphic function, $\Gamma=$ $\left\{\left(k_{1}, j_{1}\right), \cdots,\left(k_{q}, j_{q}\right)\right\}$ be a finite set of distinct pairs of integers such that $k_{i}>$ $j_{i} \geq 0, i=1, \cdots, q, \alpha(>1)$ be a given real constant. Then there exists a subset $E \subset(1,+\infty)$ with finite logarithmic measure, and there exists a constant $B(>0)$ that depend only on $\alpha$ and $\Gamma$, such that for all $z$ satisfying $|z| \notin[0,1] \cup E$ and for all $(k, j) \in \Gamma$, we have

$$
\left|\frac{f^{(k)}(z)}{f^{(j)}(z)}\right| \leq B\left(\frac{T(\alpha r, f)}{r} \log ^{\alpha} r \log T(\alpha r, f)\right)^{k-j} ;
$$

(2) if $\sigma(f)=\sigma<\infty$, then for any given $\varepsilon(>0)$, there exists a subset $E \subset$ $(1,+\infty)$ with finite logarithmic measure such that for all $z$ satisfying $|z| \notin[0,1] \cup E$ 
Zhen Chen, Xiu-Min Zheng - Meromorphic solutions of differential-difference ...

and for all $(k, j) \in \Gamma$, we have

$$
\left|\frac{f^{(k)}(z)}{f^{(j)}(z)}\right| \leq|z|^{(k-j)(\sigma-1+\varepsilon)} .
$$

Lemma 9. (see[3]) Let $f(z)$ be a meromorphic function, $c$ is a non-zero complex constant. Then the following inequalities

$$
(1+o(1)) T(r-|c|, f) \leq T(r, f(z+c)) \leq(1+o(1)) T(r+|c|, f)
$$

hold as $r \rightarrow \infty$. Therefore, it is easy to obtain that

$$
\sigma(f(z+c))=\sigma(f), \quad \mu(f(z+c))=\mu(f) .
$$

Lemma 10. (see[2]) Let $\eta_{1}, \eta_{2}$ be distinct complex numbers, and let $f(z)$ be a finite order meromorphic function. Let $\sigma$ be the order of $f(z)$. Then for each $\varepsilon(>0)$, we have

$$
m\left(r, \frac{f\left(z+\eta_{1}\right)}{f\left(z+\eta_{2}\right)}\right)=O\left(r^{\sigma-1+\varepsilon}\right) .
$$

The following two lemmas are the special case $p=1$ of the original ones in $[6]$ and [12] respectively.

Lemma 11. (see[6]) Let $f(z)$ be an entire function with $\mu(f)<\infty$, then for any given $\varepsilon(>0)$, there exists a subset $H \subset(1,+\infty)$ with infinite logarithmic measure such that for all $r \in H$, we have

$$
\mu(f)=\lim _{\substack{r \rightarrow \infty \\ r \in H}} \frac{\log \log M(r, f)}{\log r} \quad \text { and } \quad M(r, f)<\exp \left\{r^{\mu(f)+\varepsilon}\right\} .
$$

Lemma 12. (see[12]) Let $f(z)$ be an entire function with $0<\mu(f)<\infty$, then for any given $\varepsilon(>0)$, there exists a subset $H \subset(1,+\infty)$ with infinite logarithmic measure such that for all $r \in H$, we have

$$
\underline{\tau}_{M}(f)=\lim _{\substack{r \rightarrow \infty \\ r \in H}} \frac{\log M(r, f)}{r^{\mu(f)}} \quad \text { and } \quad M(r, f)<\exp \left\{\left(\underline{\tau}_{M}(f)+\varepsilon\right) r^{\mu(f)}\right\} .
$$

We also have the similar two lemmas for the case of meromorphic functions.

Lemma 13. (see[14]) Let $f(z)$ be a meromorphic function with $\mu(f)<\infty$. Then for any given $\varepsilon(>0)$, there exists a subset $H \subset(1,+\infty)$ with infinite logarithmic measure such that for all $r \in H$, we have

$$
T(r, f)<r^{\mu(f)+\varepsilon} \text {. }
$$

Lemma 14. (see[9]) Let $f(z)$ be a meromorphic function with $0<\mu(f)<\infty$. Then for any given $\varepsilon(>0)$, there exists a subset $H \subset(1,+\infty)$ with infinite logarithmic measure such that for all $r \in H$, we have

$$
T(r, f)<(\underline{\tau}(f)+\varepsilon) r^{\mu(f)} .
$$


Zhen Chen, Xiu-Min Zheng - Meromorphic solutions of differential-difference ...

\section{Proofs of Theorems 5 And 6}

\subsection{Proof of Theorem 5}

Suppose that $f(z)$ is a non-zero meromorphic solution of (3). If $f(z)$ has infinite order, the result holds yet. Then we assume that $f(z)$ has finite order without loss of generality.

We divide (3) by $f\left(z+c_{l}\right)$ to get

$$
-A_{l 0}(z)=\sum_{\substack{i=0 \\ i \neq l}}^{n} \sum_{j=0}^{m} A_{i j}(z) \frac{f^{(j)}\left(z+c_{i}\right)}{f\left(z+c_{i}\right)} \frac{f\left(z+c_{i}\right)}{f\left(z+c_{l}\right)}+\sum_{j=1}^{m} A_{l j}(z) \frac{f^{(j)}\left(z+c_{l}\right)}{f\left(z+c_{l}\right)}
$$

consequently

$$
\begin{aligned}
\left|A_{l 0}(z)\right| \leq & \sum_{\substack{i=0 \\
i \neq l}}^{n} \sum_{j=0}^{m}\left|A_{i j}(z)\right|\left|\frac{f^{(j)}\left(z+c_{i}\right)}{f\left(z+c_{i}\right)}\right|\left|\frac{f\left(z+c_{i}\right)}{f\left(z+c_{l}\right)}\right| \\
& +\sum_{j=1}^{m}\left|A_{l j}(z)\right|\left|\frac{f^{(j)}\left(z+c_{l}\right)}{f\left(z+c_{l}\right)}\right| .
\end{aligned}
$$

It follows by Lemma 7 that for any given $\varepsilon(>0)$, there exists a subset $E_{1} \subset$ $(1,+\infty)$ with finite logarithmic measure such that for all $|z|=r \notin[0,1] \cup E_{1}$, we have

$$
\left|\frac{f\left(z+c_{i}\right)}{f\left(z+c_{l}\right)}\right| \leq \exp \left\{r^{\sigma(f)-1+\varepsilon}\right\}, \quad i \neq l
$$

It follows by Lemmas 8 and 9 that for the above $\varepsilon$, there exists a subset $E_{2} \subset(1,+\infty)$ with finite logarithmic measure such that for all $|z|=r \notin[0,1] \cup E_{2}$, we have

$$
\left|\frac{f^{(j)}\left(z+c_{i}\right)}{f\left(z+c_{i}\right)}\right| \leq r^{j\left(\sigma\left(f\left(z+c_{i}\right)\right)-1+\varepsilon\right)}=r^{j(\sigma(f)-1+\varepsilon)}, \quad(i, j) \neq(l, 0) .
$$

In the following, we divide the proof into four cases.

Case (i) We suppose that $\sigma<\mu\left(A_{l 0}\right)$.

By the definitions of $\sigma\left(A_{i j}\right),(i, j) \neq(a, b),(l, 0)$, we have for the above $\varepsilon$ and sufficiently large $r$,

$$
M\left(r, A_{i j}\right) \leq \exp \left\{r^{\sigma\left(A_{i j}\right)+\varepsilon}\right\} \leq \exp \left\{r^{\sigma+\varepsilon}\right\}, \quad(i, j) \neq(a, b),(l, 0) .
$$

By the definition of $\mu\left(A_{l 0}\right)$, we have for sufficiently small $\varepsilon$ and sufficiently large $r$,

$$
M\left(r, A_{l 0}\right) \geq \exp \left\{r^{\mu\left(A_{l 0}\right)-\varepsilon}\right\} .
$$


Zhen Chen, Xiu-Min Zheng - Meromorphic solutions of differential-difference ...

By the definition of $\mu\left(A_{a b}\right)$ and Lemma 11 , there exists a subset $H_{1} \subset(1,+\infty)$ with infinite logarithmic measure such that for all $r \in H_{1}$, we have

$$
M\left(r, A_{a b}\right) \leq \exp \left\{r^{\mu\left(A_{a b}\right)+\varepsilon}\right\} .
$$

Then for all $z$ satisfying $|z|=r \in H_{1} \backslash\left([0,1] \cup E_{1} \cup E_{2}\right), r \rightarrow \infty$ and $\left|A_{l 0}(z)\right|=$ $M\left(r, A_{l 0}\right)$, we deduce from (5)-(10) that

$$
\begin{aligned}
& \exp \left\{r^{\mu\left(A_{l 0}\right)-\varepsilon}\right\} \\
\leq & {\left[\exp \left\{r^{\sigma+2 \varepsilon}\right\}+\exp \left\{r^{\mu\left(A_{a b}\right)+\varepsilon}\right\}\right] \exp \left\{r^{\sigma(f)-1+\varepsilon}\right\} r^{m(\sigma(f)-1+\varepsilon)} . }
\end{aligned}
$$

Now, we may choose sufficiently small $\varepsilon$ satisfying $0<3 \varepsilon<\mu\left(A_{l 0}\right)-\sigma$ and deduce from (11) that for $r \in H_{1} \backslash\left([0,1] \cup E_{1} \cup E_{2}\right), r \rightarrow \infty$,

$$
\exp \left\{r^{\mu\left(A_{l 0}\right)-2 \varepsilon}\right\} \leq \exp \left\{r^{\sigma(f)-1+\varepsilon}\right\}
$$

that is, $\sigma(f) \geq \mu\left(A_{l 0}\right)+1-3 \varepsilon$. Since $\varepsilon$ is arbitrary, we have $\sigma(f) \geq \mu\left(A_{l 0}\right)+1$.

Case (ii) We suppose that $\max \left\{\sigma\left(A_{i j}\right),(i, j) \neq(a, b),(l, 0)\right\}=\alpha<\mu\left(A_{a b}\right)=$ $\mu\left(A_{l 0}\right)$ and $\underline{\tau}_{M}\left(A_{l 0}\right)>\underline{\tau}_{M}\left(A_{a b}\right)$.

By the definitions of $\sigma\left(A_{i j}\right),(i, j) \neq(a, b),(l, 0)$, we have for the above $\varepsilon$ and sufficiently large $r$,

$$
M\left(r, A_{i j}\right) \leq \exp \left\{r^{\sigma\left(A_{i j}\right)+\varepsilon}\right\} \leq \exp \left\{r^{\alpha+\varepsilon}\right\}, \quad(i, j) \neq(a, b),(l, 0) .
$$

By the definition of $\underline{\tau}_{M}\left(A_{l 0}\right)$, we have for sufficiently small $\varepsilon$ and sufficiently large $r$,

$$
M\left(r, A_{l 0}\right) \geq \exp \left\{\left(\underline{\tau}_{M}\left(A_{l 0}\right)-\varepsilon\right) r^{\mu\left(A_{l 0}\right)}\right\} .
$$

By the definition of $\underline{\tau}_{M}\left(A_{a b}\right)$ and Lemma 12, there exists a subset $H_{2} \subset(1,+\infty)$ with infinite logarithmic measure such that for all $r \in H_{2}$, we have

$$
M\left(r, A_{a b}\right) \leq \exp \left\{\left(\underline{\tau}_{M}\left(A_{a b}\right)+\varepsilon\right) r^{\mu\left(A_{a b}\right)}\right\}=\exp \left\{\left(\underline{\tau}_{M}\left(A_{a b}\right)+\varepsilon\right) r^{\mu\left(A_{l 0}\right)}\right\} .
$$

Then for all $z$ satisfying $|z|=r \in H_{2} \backslash\left([0,1] \cup E_{1} \cup E_{2}\right), r \rightarrow \infty$ and $\left|A_{l 0}(z)\right|=$ $M\left(r, A_{l 0}\right)$, we deduce from (5)-(7), (12)-(14) that

$$
\begin{aligned}
\exp \left\{\left(\underline{\tau}_{M}\left(A_{l 0}\right)-\varepsilon\right) r^{\mu\left(A_{l 0}\right)}\right\} \leq & {\left[\exp \left\{r^{\alpha+2 \varepsilon}\right\}+\exp \left\{\left(\underline{\tau}_{M}\left(A_{a b}\right)+\varepsilon\right) r^{\mu\left(A_{l 0}\right)}\right\}\right] } \\
& \exp \left\{r^{\sigma(f)-1+\varepsilon}\right\} r^{m(\sigma(f)-1+\varepsilon)} .
\end{aligned}
$$

Now, we may choose sufficiently small $\varepsilon$ satisfying $0<3 \varepsilon<\min \left\{\mu\left(A_{l 0}\right)-\right.$ $\left.\alpha, \underline{\tau}_{M}\left(A_{l 0}\right)-\underline{\tau}_{M}\left(A_{a b}\right)\right\}$ and deduce from (15) that for $r \in H_{2} \backslash\left([0,1] \cup E_{1} \cup E_{2}\right)$, $r \rightarrow \infty$,

$$
\exp \left\{\left(\underline{\tau}_{M}\left(A_{l 0}\right)-\underline{\tau}_{M}\left(A_{a b}\right)-3 \varepsilon\right) r^{\mu\left(A_{l 0}\right)}\right\} \leq \exp \left\{r^{\sigma(f)-1+\varepsilon}\right\}
$$


Zhen Chen, Xiu-Min Zheng - Meromorphic solutions of differential-difference ...

that is, $\sigma(f) \geq \mu\left(A_{l 0}\right)+1-\varepsilon$. Since $\varepsilon$ is arbitrary, we have $\sigma(f) \geq \mu\left(A_{l 0}\right)+1$.

Case (iii) We suppose that $\mu\left(A_{a b}\right)<\max \left\{\sigma\left(A_{i j}\right),(i, j) \neq(a, b),(l, 0)\right\}=\mu\left(A_{l 0}\right)$ and $\underline{\tau}_{M}\left(A_{l 0}\right)>\tau_{1}=\max \left\{\tau_{M}\left(A_{i j}\right): \sigma\left(A_{i j}\right)=\mu\left(A_{l 0}\right),(i, j) \neq(a, b),(l, 0)\right\}$.

By the definitions of $\sigma\left(A_{i j}\right)$ and $\tau_{M}\left(A_{i j}\right),(i, j) \neq(a, b),(l, 0)$, we have for sufficiently small $\varepsilon$ and sufficiently large $r$,

$$
M\left(r, A_{i j}\right) \leq \begin{cases}\exp \left\{r^{\mu\left(A_{l 0}\right)-\varepsilon}\right\}, & \text { if } \sigma\left(A_{i j}\right)<\mu\left(A_{l 0}\right),(i, j) \neq(a, b),(l, 0) \\ \exp \left\{\left(\tau_{1}+\varepsilon\right) r^{\mu\left(A_{l 0}\right)}\right\}, & \text { if } \sigma\left(A_{i j}\right)=\mu\left(A_{l 0}\right),(i, j) \neq(a, b),(l, 0) .\end{cases}
$$

Then for all $z$ satisfying $|z|=r \in H_{1} \backslash\left([0,1] \cup E_{1} \cup E_{2}\right), r \rightarrow \infty$ and $\left|A_{l 0}(z)\right|=$ $M\left(r, A_{l 0}\right)$, we deduce from (5)-(7), (10), (13) and (16) that

$$
\begin{aligned}
& \exp \left\{\left(\underline{\tau}_{M}\left(A_{l 0}\right)-\varepsilon\right) r^{\mu\left(A_{l 0}\right)}\right\} \\
\leq & {\left[\exp \left\{r^{\mu\left(A_{l 0}\right)-\frac{\varepsilon}{2}}\right\}+\exp \left\{r^{\mu\left(A_{a b}\right)+\varepsilon}\right\}+\exp \left\{\left(\tau_{1}+2 \varepsilon\right) r^{\mu\left(A_{l 0}\right)}\right\}\right] } \\
& \exp \left\{r^{\sigma(f)-1+\varepsilon}\right\} r^{m(\sigma(f)-1+\varepsilon)} .
\end{aligned}
$$

Now, we may choose sufficiently small $\varepsilon$ satisfying $0<4 \varepsilon<\min \left\{\mu\left(A_{l 0}\right)-\mu\left(A_{a b}\right)\right.$, $\left.\underline{\tau}_{M}\left(A_{l 0}\right)-\tau_{1}\right\}$ and deduce from (17) that for $r \in H_{1} \backslash\left([0,1] \cup E_{1} \cup E_{2}\right), r \rightarrow \infty$,

$$
\exp \left\{\left(\underline{\tau}_{M}\left(A_{l 0}\right)-\tau_{1}-4 \varepsilon\right) r^{\mu\left(A_{l 0}\right)}\right\} \leq \exp \left\{r^{\sigma(f)-1+\varepsilon}\right\},
$$

that is, $\sigma(f) \geq \mu\left(A_{l 0}\right)+1-\varepsilon$. Since $\varepsilon$ is arbitrary, we have $\sigma(f) \geq \mu\left(A_{l 0}\right)+1$.

Case (iv) We suppose that $\max \left\{\sigma\left(A_{i j}\right),(i, j) \neq(a, b),(l, 0)\right\}=\mu\left(A_{a b}\right)=\mu\left(A_{l 0}\right)$ and $\underline{\tau}_{M}\left(A_{l 0}\right)>\tau_{2}=\max \left\{\underline{\tau}_{M}\left(A_{a b}\right), \tau_{M}\left(A_{i j}\right): \sigma\left(A_{i j}\right)=\mu\left(A_{l 0}\right),(i, j) \neq(a, b),(l, 0)\right\}$.

Then for all $z$ satisfying $|z|=r \in H_{2} \backslash\left([0,1] \cup E_{1} \cup E_{2}\right), r \rightarrow \infty$ and $\left|A_{l 0}(z)\right|=$ $M\left(r, A_{l 0}\right)$, we deduce from (5)-(7), (13), (14) and (16) that

$$
\begin{aligned}
\exp \left\{\left(\underline{\tau}_{M}\left(A_{l 0}\right)-\varepsilon\right) r^{\mu\left(A_{l 0}\right)}\right\} \leq & {\left[\exp \left\{\left(\tau_{2}+2 \varepsilon\right) r^{\mu\left(A_{l 0}\right)}\right\}+\exp \left\{r^{\mu\left(A_{l 0}\right)-\frac{\varepsilon}{2}}\right\}\right] } \\
& \exp \left\{r^{\sigma(f)-1+\varepsilon}\right\} r^{m(\sigma(f)-1+\varepsilon)} .
\end{aligned}
$$

Now, we may choose sufficiently small $\varepsilon$ satisfying $0<4 \varepsilon<\underline{\tau}_{M}\left(A_{l 0}\right)-\tau_{2}$ and deduce from (18) that for $r \in H_{2} \backslash\left([0,1] \cup E_{1} \cup E_{2}\right), r \rightarrow \infty$,

$$
\exp \left\{\left(\underline{\tau}_{M}\left(A_{l 0}\right)-\tau_{2}-4 \varepsilon\right) r^{\mu\left(A_{l 0}\right)}\right\} \leq \exp \left\{r^{\sigma(f)-1+\varepsilon}\right\},
$$

that is, $\sigma(f) \geq \mu\left(A_{l 0}\right)+1-\varepsilon$. Since $\varepsilon$ is arbitrary, we have $\sigma(f) \geq \mu\left(A_{l 0}\right)+1$.

Therefore, the proof of Theorem 5 is complete.

\subsection{Proof of Theorem 6}

Suppose that $f(z)$ is a non-zero meromorphic solution of (3). If $f(z)$ has infinite order, the result holds yet. Then we assume that $f(z)$ has finite order without loss of generality. 
Zhen Chen, Xiu-Min Zheng - Meromorphic solutions of differential-difference ...

It follows by (4) that

$$
\begin{aligned}
m\left(r, A_{l 0}\right) \leq & \sum_{\substack{i=0 \\
i \neq l}}^{n} \sum_{j=0}^{m} m\left(r, A_{i j}\right)+\sum_{j=1}^{m} m\left(r, A_{l j}\right)+\sum_{\substack{i=0 \\
i \neq l}}^{n} m\left(r, \frac{f\left(z+c_{i}\right)}{f\left(z+c_{l}\right)}\right) \\
& +\sum_{j=1}^{m} \sum_{i=0}^{n} m\left(r, \frac{f^{(j)}\left(z+c_{i}\right)}{f\left(z+c_{i}\right)}\right)+O(1) .
\end{aligned}
$$

It follows by Lemmas 9 and 10 and the lemma of logarithmic derivative that for any given $\varepsilon(>0)$, we have

$$
\begin{aligned}
& m\left(r, \frac{f\left(z+c_{i}\right)}{f\left(z+c_{l}\right)}\right)=O\left(r^{\sigma(f)-1+\varepsilon}\right), \quad i \neq l, \\
& m\left(r, \frac{f^{(j)}\left(z+c_{i}\right)}{f\left(z+c_{i}\right)}\right)=O(\log r), \quad j \neq 0 .
\end{aligned}
$$

In the following, we divide the proof into four cases.

Case (i) We suppose that $\sigma<\mu\left(A_{l 0}\right)$.

By the definitions of $\sigma\left(A_{i j}\right),(i, j) \neq(a, b),(l, 0)$, we have for the above $\varepsilon$ and sufficiently large $r$,

$$
m\left(r, A_{i j}\right) \leq T\left(r, A_{i j}\right) \leq r^{\sigma\left(A_{i j}\right)+\varepsilon}, \quad(i, j) \neq(a, b),(l, 0) .
$$

By the definition of $\mu\left(A_{l 0}\right)$ and the assumption $\delta\left(\infty, A_{l 0}\right)=\delta>0$, we have for sufficiently small $\varepsilon$ and sufficiently large $r$,

$$
m\left(r, A_{l 0}\right) \geq \frac{\delta}{2} T\left(r, A_{l 0}\right) \geq \frac{\delta}{2} r^{\mu\left(A_{l 0}\right)-\frac{\varepsilon}{2}} \geq r^{\mu\left(A_{l 0}\right)-\varepsilon} .
$$

By the definition of $\mu\left(A_{a b}\right)$ and Lemma 13, there exists a subset $H_{1} \subset(1,+\infty)$ with infinite logarithmic measure such that for all $r \in H_{1}$, we have

$$
m\left(r, A_{a b}\right) \leq T\left(r, A_{a b}\right) \leq r^{\mu\left(A_{a b}\right)+\varepsilon} .
$$

Then it follows by (19)-(23) that for sufficiently large $r \in H_{1}$, we have

$$
\begin{aligned}
r^{\mu\left(A_{l 0}\right)-\varepsilon} & \leq r^{\mu\left(A_{a b}\right)+\varepsilon}+O\left(r^{\sigma+\varepsilon}\right)+O\left(r^{\sigma(f)-1+\varepsilon}\right)+O(\log r) \\
& \leq r^{\sigma+2 \varepsilon}+r^{\sigma(f)-1+2 \varepsilon} .
\end{aligned}
$$

Now, we may choose sufficiently small $\varepsilon$ satisfying $0<3 \varepsilon<\mu\left(A_{l 0}\right)-\sigma$ and deduce from (24) that for sufficiently large $r \in H_{1}$,

$$
r^{\mu\left(A_{l 0}\right)-2 \varepsilon} \leq r^{\sigma(f)-1+2 \varepsilon}
$$


Zhen Chen, Xiu-Min Zheng - Meromorphic solutions of differential-difference ...

that is, $\sigma(f) \geq \mu\left(A_{l 0}\right)+1-4 \varepsilon$. Since $\varepsilon$ is arbitrary, we have $\sigma(f) \geq \mu\left(A_{l 0}\right)+1$.

Case (ii) We suppose that $\max \left\{\sigma\left(A_{i j}\right),(i, j) \neq(a, b),(l, 0)\right\}=\alpha<\mu\left(A_{a b}\right)=$ $\mu\left(A_{l 0}\right)$ and $\delta \underline{\tau}\left(A_{l 0}\right)>\underline{\tau}\left(A_{a b}\right)$.

By the definition of $\underline{\tau}\left(A_{l 0}\right)$ and the assumption $\delta\left(\infty, A_{l 0}\right)=\delta>0$, we have for sufficiently small $\varepsilon$ and sufficiently large $r$,

$$
\begin{aligned}
m\left(r, A_{l 0}\right) & \geq(\delta-\varepsilon) T\left(r, A_{l 0}\right) \geq(\delta-\varepsilon)\left(\underline{\tau}\left(A_{l 0}\right)-\varepsilon\right) r^{\mu\left(A_{l 0}\right)} \\
& \geq\left(\delta \underline{\tau}\left(A_{l 0}\right)-\left(\underline{\tau}\left(A_{l 0}\right)+1\right) \varepsilon\right) r^{\mu\left(A_{l 0}\right)} .
\end{aligned}
$$

By the definition of $\underline{\tau}\left(A_{a b}\right)$ and Lemma 14, there exists a subset $H_{2} \subset(1,+\infty)$ with infinite logarithmic measure such that for all $r \in H_{2}$, we have

$$
m\left(r, A_{a b}\right) \leq T\left(r, A_{a b}\right) \leq\left(\underline{\tau}\left(A_{a b}\right)+\varepsilon\right) r^{\mu\left(A_{a b}\right)}=\left(\underline{\tau}\left(A_{a b}\right)+\varepsilon\right) r^{\mu\left(A_{l 0}\right)} .
$$

Then it follows by (19)-(21), (25) and (26) that for sufficiently large $r \in H_{2}$, we have

$$
\begin{aligned}
& \left(\delta \underline{\tau}\left(A_{l 0}\right)-\left(\underline{\tau}\left(A_{l 0}\right)+1\right) \varepsilon\right) r^{\mu\left(A_{l 0}\right)} \\
\leq & \left(\underline{\tau}\left(A_{a b}\right)+\varepsilon\right) r^{\mu\left(A_{l 0}\right)}+O\left(r^{\alpha+\varepsilon}\right)+O\left(r^{\sigma(f)-1+\varepsilon}\right)+O(\log r) \\
\leq & \left(\underline{\tau}\left(A_{a b}\right)+\varepsilon\right) r^{\mu\left(A_{l 0}\right)}+r^{\alpha+2 \varepsilon}+r^{\sigma(f)-1+2 \varepsilon}
\end{aligned}
$$

Now, we may choose sufficiently small $\varepsilon$ satisfying $0<\left(\underline{\tau}\left(A_{l 0}\right)+3\right) \varepsilon<\min \left\{\mu\left(A_{l 0}\right)-\right.$ $\left.\alpha, \delta \underline{\tau}\left(A_{l 0}\right)-\underline{\tau}\left(A_{a b}\right)\right\}$ and deduce from (27) that for sufficiently large $r \in H_{2}$,

$$
\left(\delta \underline{\tau}\left(A_{l 0}\right)-\underline{\tau}\left(A_{a b}\right)-\left(\underline{\tau}\left(A_{l 0}\right)+3\right) \varepsilon\right) r^{\mu\left(A_{l 0}\right)} \leq r^{\sigma(f)-1+2 \varepsilon},
$$

that is, $\sigma(f) \geq \mu\left(A_{l 0}\right)+1-2 \varepsilon$. Since $\varepsilon$ is arbitrary, we have $\sigma(f) \geq \mu\left(A_{l 0}\right)+1$.

Case (iii) We suppose that $\mu\left(A_{a b}\right)<\max \left\{\sigma\left(A_{i j}\right),(i, j) \neq(a, b),(l, 0)\right\}=\mu\left(A_{l 0}\right)$ and $\delta \underline{\tau}\left(A_{l 0}\right)>\tau_{1}=\max \left\{\tau\left(A_{i j}\right): \sigma\left(A_{i j}\right)=\mu\left(A_{l 0}\right),(i, j) \neq(a, b),(l, 0)\right\}$.

By the definitions of $\sigma\left(A_{i j}\right)$ and $\tau\left(A_{i j}\right),(i, j) \neq(a, b),(l, 0)$, we have for sufficiently small $\varepsilon$ and sufficiently large $r$,

$$
T\left(r, A_{i j}\right) \leq \begin{cases}r^{\mu\left(A_{l 0}\right)-\varepsilon}, & \text { if } \sigma\left(A_{i j}\right)<\mu\left(A_{l 0}\right),(i, j) \neq(a, b),(l, 0) ; \\ \left(\tau_{1}+\varepsilon\right) r^{\mu\left(A_{l 0}\right)}, & \text { if } \sigma\left(A_{i j}\right)=\mu\left(A_{l 0}\right),(i, j) \neq(a, b),(l, 0) .\end{cases}
$$

Then it follows by (19), (20), (23), (25) and (28) that for sufficiently large $r \in H_{1}$, we have

$$
\begin{aligned}
& \left(\delta \underline{\tau}\left(A_{l 0}\right)-\left(\underline{\tau}\left(A_{l 0}\right)+1\right) \varepsilon\right) r^{\mu\left(A_{l 0}\right)} \\
\leq & r^{\mu\left(A_{a b}\right)+\varepsilon}+O\left(\left(\tau_{1}+\varepsilon\right) r^{\mu\left(A_{l 0}\right)}\right)+O\left(r^{\mu\left(A_{l 0}\right)-\varepsilon}\right)+O\left(r^{\sigma(f)-1+\varepsilon}\right)+O(\log r) \\
\leq & r^{\mu\left(A_{a b}\right)+\varepsilon}+\left(\tau_{1}+2 \varepsilon\right) r^{\mu\left(A_{l 0}\right)}+r^{\sigma(f)-1+2 \varepsilon}
\end{aligned}
$$


Zhen Chen, Xiu-Min Zheng - Meromorphic solutions of differential-difference ...

Now, we may choose sufficiently small $\varepsilon$ satisfying $0<\left(\underline{\tau}\left(A_{l 0}\right)+3\right) \varepsilon<\min \left\{\mu\left(A_{l 0}\right)-\right.$ $\left.\mu\left(A_{a b}\right), \delta \underline{\tau}\left(A_{l 0}\right)-\tau_{1}\right\}$ and deduce from (29) that for sufficiently large $r \in H_{1}$,

$$
\left(\delta \underline{\tau}\left(A_{l 0}\right)-\tau_{1}-\left(\underline{\tau}\left(A_{l 0}\right)+3\right) \varepsilon\right) r^{\mu\left(A_{l 0}\right)} \leq r^{\sigma(f)-1+2 \varepsilon},
$$

that is, $\sigma(f) \geq \mu\left(A_{l 0}\right)+1-2 \varepsilon$. Since $\varepsilon$ is arbitrary, we have $\sigma(f) \geq \mu\left(A_{l 0}\right)+1$.

Case (iv) We suppose that $\max \left\{\sigma\left(A_{i j}\right),(i, j) \neq(a, b),(l, 0)\right\}=\mu\left(A_{a b}\right)=\mu\left(A_{l 0}\right)$ and $\delta \underline{\tau}\left(A_{l 0}\right)>\tau_{2}=\max \left\{\underline{\tau}\left(A_{a b}\right), \tau\left(A_{i j}\right): \sigma\left(A_{i j}\right)=\mu\left(A_{l 0}\right),(i, j) \neq(a, b),(l, 0)\right\}$.

Then it follows by (19), (20), (25), (26) and (28) that for sufficiently large $r \in H_{2}$, we have

$$
\begin{aligned}
& \left(\delta \underline{\tau}\left(A_{l 0}\right)-\left(\underline{\tau}\left(A_{l 0}\right)+1\right) \varepsilon\right) r^{\mu\left(A_{l 0}\right)} \\
\leq & O\left(\left(\tau_{2}+\varepsilon\right) r^{\mu\left(A_{l 0}\right)}\right)+O\left(r^{\mu\left(A_{l 0}\right)-\varepsilon}\right)+O\left(r^{\sigma(f)-1+\varepsilon}\right)+O(\log r) \\
\leq & \left(\tau_{2}+2 \varepsilon\right) r^{\mu\left(A_{l 0}\right)}+r^{\sigma(f)-1+2 \varepsilon} .
\end{aligned}
$$

Now, we may choose sufficiently small $\varepsilon$ satisfying $0<\left(\underline{\tau}\left(A_{l 0}\right)+3\right) \varepsilon<\delta \underline{\tau}\left(A_{l 0}\right)-\tau_{2}$ and deduce from (30) that for sufficiently large $r \in H_{2}$,

$$
\left(\delta \underline{\tau}\left(A_{l 0}\right)-\tau_{2}-\left(\underline{\tau}\left(A_{l 0}\right)+3\right) \varepsilon\right) r^{\mu\left(A_{l 0}\right)} \leq r^{\sigma(f)-1+2 \varepsilon},
$$

that is, $\sigma(f) \geq \mu\left(A_{l 0}\right)+1-2 \varepsilon$. Since $\varepsilon$ is arbitrary, we have $\sigma(f) \geq \mu\left(A_{l 0}\right)+1$.

Therefore, the proof of Theorem 6 is complete.

Acknowledgements. The authors are grateful to the referees and editors for their valuable comments which lead to the improvement of this paper. This project was supported by the National Natural Science Foundation of China (11761035) and the Natural Science Foundation of Jiangxi Province in China (20171BAB201002).

\section{REFERENCES}

[1] B. Belaïdi, Growth and oscillation of solutions to linear differential equations with entire coefficients having the same order, Electron. J. Differ. Equ. 70 (2009), $1-10$.

[2] Y. M. Chiang, S. J. Feng, On the Nevanlinna characteristic of $f(z+\eta)$ and difference equations in the complex plane, Ramanujan J. 16 (2008), 105-129.

[3] A. A. Goldberg, I. V. Ostrovskii, The distribution of values of meromorphic functions, Nauka, Moscow, 1970. (in Russian)

[4] G. G. Gundersen, Estimates for the logarithmic derivative of a meromoephic function, plus similar estimates, J. London Math. Soc. 37, 2 (1988), 88-104.

[5] W. K. Hayman, Meromorphic functions, Clarendon Press, Oxford, 1964. 
Zhen Chen, Xiu-Min Zheng - Meromorphic solutions of differential-difference ...

[6] H. Hu, X. M. Zheng, Growth of solutions to linear differential equations with entire coefficients, Electron. J. Differ. Equ. 226 (2012), 1-15.

[7] I. Laine, Nevanlinna theory and complex differential equations, Walter de Gruyter, Berlin, 1993.

[8] I. Laine, C. C. Yang, Clunie theorems for difference and q-difference polynomials, J. London Math. Soc. 76, 3 (2007), 556-566.

[9] L. Q. Luo, X. M. Zheng, Growth of meromorphic solutions of some kind of complex linear difference equation with entire or meromorphic coefficients, Mathematica Applicata. 29, 4 (2016), 723-730.

[10] L. Q. Luo, X. M. Zheng, Value distribution of meromorphic solutions of homogeneous and non-homogeneous complex linear differential-difference equations, Open Math. 14, 1 (2016), 970-976.

[11] J. Tu, G. T. Deng, Growth of solutions of higher order linear differential equations with the coefficient $A_{0}$ being dominant, Acta Math. Sci. 30, 4 (2010), 945-952. (in Chinese)

[12] S. Z. Wu, X. M. Zheng, Growth of solutions of some kind of higher-order linear differential equations in $\mathbb{C}$ and in $\Delta$, Mathematica Applicata. 29, 1 (2016), 20-30. (in Chinese)

[13] C. C. Yang, H. X. Yi, Uniqueness theory of meromorphic functions, Klumer Academic Publishers Group, Dordrecht, 2003.

[14] X. M. Zheng, J. Tu, Growth of meromorphic solutions of linear difference equations, J. Math. Anal. Appl. 384 (2011), 349-356.

[15] Y. P. Zhou, X. M. Zheng, Growth of meromorphic solutions to homogeneous and non-homogeneous linear (differential-)difference equations with meromorphic coefficients, Electron. J. Differ. Equ. 34 (2017), 1-15.

Zhen Chen

Institute of Mathematics and Information Science, Jiangxi Normal University,

Nanchang, China

email: misschenzhen@163.com

Xiu-Min Zheng (Corresponding author)

Institute of Mathematics and Information Science, Jiangxi Normal University,

Nanchang, China

email: zhengxiumin2008@sina.com 